\title{
Investigation of the existence domain for Dyakonov surface waves in the Sage computer algebra system
}

\author{
Oleg K. Kroytor \\ Peoples' Friendship University of Russia (RUDN University) \\ 6, Miklukho-Maklaya St., Moscow, 117198, Russian Federation
}

(received: March 25, 2021; accepted: May 25, 2021)

Surface electromagnetic waves (Dyakonov waves) propagating along a plane interface between an isotropic substance with a constant dielectric constant and an anisotropic crystal, whose dielectric tensor has a symmetry axis directed along the interface, are considered. It is well known that the question of the existence of such surface waves is reduced to the question of the existence of a solution to a certain system of algebraic equations and inequalities. In the present work, this system is investigated in the Sage computer algebra system.

The built-in technique of exceptional ideals in Sage made it possible to describe the solution of a system of algebraic equations parametrically using a single parameter, with all the original quantities expressed in terms of this parameter using radicals. The remaining inequalities were only partially investigated analytically. For a complete study of the solvability of the system of equations and inequalities, a symbolicnumerical algorithm is proposed and implemented in Sage, and the results of computer experiments are presented. Based on these results, conclusions were drawn that require further theoretical substantiation.

Key words and phrases: surface waves, Dyakonov waves, electromagnetic waves, computer algebra, Sage

\section{Introduction}

In the 1980s, a special class of solutions to Maxwell's equations was theoretically discovered, namely, electromagnetic waves traveling along the interface between two dielectrics, the intensity of which rapidly decreases with distance from the interface [1]-[6]. These waves are called Dyakonov surface waves. Experimental observation of surface waves was carried out quite recently [7], [8]. In theoretical works, as in the work of Dyakonov itself [2], the question of the existence of surface waves was reduced to the question of the existence of a solution to a certain system of algebraic equations and inequalities that cannot be solved analytically, which hinders further research. In this paper, it will be shown what computer algebra systems can give for these systems.

(C) Kroytor O.K., 2021

This work is licensed under a Creative Commons Attribution 4.0 International License

http://creativecommons.org/licenses/by/4.0/ 


\section{Surface waves}

We investigate the classical problem of waves propagating along the interface of an anisotropic medium with permittivity

$$
\epsilon=\operatorname{diag}\left(\epsilon_{o}, \epsilon_{o}, \epsilon_{e}\right)
$$

and isotropic medium with constant permittivity $\epsilon$. For definiteness, let the plane $x=0$ serve as an interface. The field in the anisotropic medium $(x<0)$ is sought in the form

$$
\begin{aligned}
& \vec{E}=\left(a_{o} \vec{E}_{o} e^{p_{o} x}+a_{e} \vec{E}_{e} e^{p_{e} x}\right) e^{i k_{y} y+i k_{z} z-i \omega t}, \\
& \vec{H}=\left(a_{o} \vec{H}_{o} e^{p_{o} x}+a_{e} \vec{H}_{e} e^{p_{e} x}\right) e^{i k_{y} y+i k_{z} z-i \omega t} .
\end{aligned}
$$

Here $\omega$ is the circular frequency of the wave, $k_{0}=\omega / c$ is the wave number, $\vec{k}_{\perp}=\left(0, k_{y}, k_{z}\right)$ is its wave vector, $a_{o}, a_{e}$ are the amplitudes of two partial waves, and positive numbers $p_{o}, p_{e}$ characterize the rate of wave decay in the anisotropic medium. Maxwell's equations give

$$
\begin{gathered}
p_{o}^{2}=k_{y}^{2}+k_{z}^{2}-\epsilon_{o} k_{0}^{2}, \\
p_{e}^{2}=k_{y}^{2}+\frac{\epsilon_{e}}{\epsilon_{o}} k_{z}^{2}-\epsilon_{e} k_{0}^{2}
\end{gathered}
$$

and for the vectors $\vec{E}_{o}, \ldots, \vec{H}_{e}$, explicit expressions are obtained, which we will not present here.

For the isotropic medium $(x>0)$ the field is described by similar formulas

$$
\begin{aligned}
& \vec{E}=\left(b_{o} \vec{E}_{o}^{\prime}+b_{e} \vec{E}_{e}^{\prime}\right) e^{-p x} e^{i k_{y} y+i k_{z} z-i \omega t}, \\
& \vec{H}=\left(b_{o} \vec{H}_{o}^{\prime}+b_{e} \vec{H}_{e}^{\prime}\right) e^{-p x} e^{i k_{y} y+i k_{z} z-i \omega t},
\end{aligned}
$$

but now the constant $p$, which characterizes the field decrease in the isotropic medium, turns out to be the same:

$$
p^{2}=k_{y}^{2}+k_{z}^{2}-\epsilon k_{0}^{2} .
$$

The conditions for matching electromagnetic fields at the interface lead to a system of homogeneous linear equations for the amplitudes $a_{o}, a_{e}, b_{o}, b_{e}$. The condition of zero determinant of this system gives the equation

$$
\begin{aligned}
\left(\left(k_{z}^{2}-\epsilon k_{0}^{2}\right) p_{o}+\left(k_{z}^{2}-\epsilon_{o} k_{0}^{2}\right) p\right)\left(\left(k_{z}^{2}-\epsilon k_{0}^{2}\right) \epsilon_{o} p_{e}+\left(k_{z}^{2}-\epsilon_{o} k_{0}^{2}\right) \epsilon p\right) & = \\
& =\left(\epsilon_{o}-\epsilon\right)^{2} k_{y}^{2} k_{z}^{2} k_{0}^{2}
\end{aligned}
$$

If real parameters $k_{y}, k_{z}, p_{o}, p_{e}$ and $p$ satisfy four algebraic equations (1), (2) and (3), then we get a solution to Maxwell's equations in the integral form defined in the entire space. These solutions exponentially decrease at $|x| \rightarrow \infty$, if the solution is in the domain 


$$
p_{o}>0, \quad p_{e}>0, \quad p>0
$$

of five-dimensional space $k_{y} k_{z} p_{o} p_{e} p$.

The existence of solution to this system does not ensure that the resulting field is not identically zero, an example will be given below. This issue requires additional check.

\section{Investigation of the system of algebraic equations}

Consider in more detail the above system of four algebraic equations (1), $(2)$, and (3).

It is possible to eliminate $k_{0}$ from this system by assuming

$$
p=k_{0} q, \quad p_{o}=k_{0} q_{o}, \quad p_{e}=k_{0} q_{e}
$$

and $k_{y}=k_{0} \beta, k_{z}=k_{0} \gamma$.

Then the system of equations is written in the form

$$
\left\{\begin{array}{l}
q_{o}^{2}=\beta^{2}+\gamma^{2}-\epsilon_{o}, \\
q_{e}^{2}=\beta^{2}+\frac{\epsilon_{e}}{\epsilon_{o}} \gamma^{2}-\epsilon_{e}, \\
q^{2}=\beta^{2}+\gamma^{2}-\epsilon, \\
\left(\left(\gamma^{2}-\epsilon\right) q_{o}+\left(\gamma^{2}-\epsilon_{o}\right) q\right)\left(\left(\gamma^{2}-\epsilon\right) \epsilon_{o} q_{e}+\left(\gamma^{2}-\epsilon_{o}\right) \epsilon q\right)= \\
=\left(\epsilon_{o}-\epsilon\right)^{2} \beta^{2} \gamma^{2} .
\end{array}\right.
$$

This is a system of 4 equations for 5 unknowns, so you can exclude 3 unknowns from it and find a connection between the remaining two. Since for applications the direction of the vector $\vec{k}_{\perp}$, that is, the ratio of $\beta$ and $\gamma$, is most interesting, it is quite natural to try to exclude the quantities $q, q_{o}$, $q_{e}$, which characterize the rate of decay of the solution with distance from surface $x=0$. However, in this way, a very complex equation is obtained, which then has to be investigated numerically.

However, it is easy to see that the unknowns $\beta$ and $\gamma$ enter the system only as squares, so it is convenient to exclude them. We did not do it by hand, but used the technique of exceptional ideals [9], implemented in the Sage computer algebra system Sage. We have eliminated the unknowns $\beta$, $\gamma, q$, and obtained an equation of the form $F\left(q_{o}, q_{e}\right)=0$, whose coefficients depend only on permittivities. The right-hand side of this equation can be represented as a product of three factors. Consider each of them separately.

First, system (5) has a solution with $q_{e}=q_{o}$. Then the difference of the first two equations of system (5) yields

$$
\frac{\epsilon_{e}-\epsilon_{o}}{\epsilon_{o}} \gamma^{2}-\left(\epsilon_{e}-\epsilon_{o}\right)=0
$$

or $\gamma^{2}=\epsilon_{o}$. 
Such a solution really exists, but on it $k_{z}^{2}-\epsilon_{o} k_{0}^{2}=0$, so that $b_{o}=b_{e}=0$ (the field in the isotropic medium is zero) and $\vec{E}_{o}$ and $\vec{E}_{e}$ become linearly dependent. Therefore, even at nonzero $a_{o}, a_{e}$ the field in the anisotropic medium can be zero. From general considerations it is obvious that it is just so in the case considered: the field cannot flow from the anisotropic medium.

The second factor yields

$$
\left(q_{e}^{2}-q_{o}^{2}\right) \epsilon_{o}=\left(\epsilon-\epsilon_{o}\right)\left(\epsilon_{e}-\epsilon_{o}\right) .
$$

The difference of first two equations of system (5) yields

$$
\left(q_{e}^{2}-q_{o}^{2}\right) \epsilon_{o}=\left(\epsilon_{e}-\epsilon_{o}\right)\left(\gamma^{2}-\epsilon_{o}\right) .
$$

Therefore, $\gamma^{2}=\epsilon$. This is the second trivial case: now $a_{o}=a_{e}=0$ and the field is absent in the anisotropic medium.

Ignoring trivial fields, we see that system (5) has a solution if and only if the third factor turns into zero:

$$
\begin{gathered}
-q_{o}^{4} \epsilon^{2}-2 q_{o}^{3} q_{e} \epsilon^{2}-q_{o}^{2} q_{e}^{2} \epsilon^{2}-2 q_{o}^{2} q_{e}^{2} \epsilon \epsilon_{o}-2 q_{o} q_{e}^{3} \epsilon \epsilon_{o}+q_{o}^{2} q_{e}^{2} \epsilon_{o}^{2}-q_{e}^{4} \epsilon_{o}^{2}+ \\
+2 q_{o}^{4} \epsilon \epsilon_{e}+2 q_{o}^{3} q_{e} \epsilon \epsilon_{e}+2 q_{o}^{3} q_{e} \epsilon_{o} \epsilon_{e}+2 q_{o}^{2} q_{e}^{2} \epsilon_{o} \epsilon_{e}- \\
-q_{e}^{2} \epsilon \epsilon_{o}^{2}+q_{e}^{2} \epsilon_{o}^{3}-2 q_{o} q_{e} \epsilon \epsilon_{o} \epsilon_{e}+2 q_{o} q_{e} \epsilon_{o}^{2} \epsilon_{e}-q_{o}^{2} \epsilon \epsilon_{e}^{2}+q_{o}^{2} \epsilon_{o} \epsilon_{e}^{2}=0 .
\end{gathered}
$$

This expression is somewhat cumbersome, however, its structure is easily seen

$$
F_{4}\left(q_{o}, q_{e}\right)+F_{2}\left(q_{o}, q_{e}\right)=0
$$

where $F_{4}, F_{2}$ are homogeneous functions of the 4-th and 2-nd order $q_{e}=t q_{o}$, so that we rewrite this equation as

$$
q_{o}^{2} F_{4}(1, t)+F_{2}(1, t)=0 .
$$

Hence

$$
q_{o}=\sqrt{\frac{-F_{2}(1, t)}{F_{4}(1, t)}},
$$

where $t$ can take any values.

The theory of exclusive ideals applied above yields an equation

$$
F\left(q_{o}, q_{e}\right)=0
$$

as a necessary and sufficient condition for the existence of a solution to system (5), but this solution can be complex and infinitely large [9]. In this case one can express the solution in terms of parameter $t$ in radicals. Thus, the investigation of solvability of the system of algebraic equations reduced to an investigation of one equation solved in radicals.

Quantities $q_{e}$ and $q$ are rather simply expressed via $t$ and $q_{o}$ : by definition

$$
q_{e}=t q_{o}
$$


and due to the first and the third equation of system (5)

$$
q^{2}=q_{o}^{2}+\epsilon_{o}-\epsilon
$$

The quantities $\beta^{2}, \gamma^{2}$ can be reconstructed by solving the system of equations linear with respect to $\beta^{2}, \gamma^{2}$

$$
\left\{\begin{array}{l}
\beta^{2}+\gamma^{2}=q_{o}^{2}+\epsilon_{o}, \\
\epsilon_{o} \beta^{2}+\epsilon_{e} \gamma^{2}=\epsilon_{o} q_{e}^{2}+\epsilon_{o} \epsilon_{e}
\end{array}\right.
$$

formed by the first and the second equation of system (5). Solving it we get

$$
\beta^{2}=\frac{\left(\epsilon_{e}-\epsilon_{o} t^{2}\right) q_{o}^{2}}{\epsilon_{e}-\epsilon_{o}}
$$

and

$$
\gamma^{2}=\frac{\epsilon_{o}\left(\epsilon_{e}-\epsilon_{o}+\left(t^{2}-1\right) q_{o}^{2}\right)}{\epsilon_{e}-\epsilon_{o}} .
$$

The issue of extracting radicals is not trivial here. From the general theory, we know that at least one choice of the root branch should result in a solution. Since only squares of $\beta$, $\gamma$ enter system (5), the solution will be obtained for any choice of signs before the radicals. However, $q$ enters the system in the first power, so that the solution of system (5) can and as we will see below will be obtained for the only choice of the branch choice for the root when calculating the value of $q$ from (7).

\section{Investigation of the system of algebraic equations and inequalities}

As was noted in [2], for the existence of surface waves the fulfillment of condition

$$
0<\epsilon_{o}<\epsilon<\epsilon_{e}
$$

is necessary. To avoid special consideration of the cases when there are deliberately no surface waves, we present here an analysis of the issue of fulfilment of inequalities

$$
q_{o}>0, \quad q_{e}>0, \quad q>0
$$

only for the Dyakonov case (10). If the first two inequalities are satisfied, then $t=q_{e} / q_{o}$ is positive. Therefore, below we restrict ourselves by considering only positive values of parameter $t$.

So, let $t$ have a positive value, and a solution of system (5) has been constructed using the formulas of the previous section. In order $q_{o}$ be positive, the fulfillment of the following inequality is necessary and sufficient:

$$
\frac{-F_{2}(1, t)}{F_{4}(1, t)}>0 \text {. }
$$


This value itself can be found from

$$
q_{o}=\sqrt{\frac{-F_{2}(1, t)}{F_{4}(1, t)}} .
$$

In this case, $q_{e}=t q_{o}$ also has a positive value.

Since $F_{2}\left(q_{o}, q_{e}\right)$ and $F_{4}\left(q_{o}, q_{e}\right)$ are terms of the second and fourth order in expression (6), we can write the radicand explicitly:

$$
F_{2}(1, t)=-\left(\epsilon_{o} t+\epsilon_{e}\right)^{2}\left(\epsilon-\epsilon_{o}\right),
$$

and

$$
F_{4}(1, t)=-\left(\epsilon_{o} t^{2}+\left(\epsilon-\epsilon_{o}\right) t+\epsilon-2 \epsilon_{e}\right)\left(\epsilon_{o} t+\epsilon\right)(t+1) .
$$

This means that in the Dyakonov case $-F_{2}>0$ and the sign of ratio $F_{2} / F_{4}$ is determined by the sign of factor

$$
\epsilon_{o} t^{2}+\left(\epsilon-\epsilon_{o}\right) t+\epsilon-2 \epsilon_{e} .
$$

The discriminant of this quadratic trinomial equals

$$
D=\epsilon^{2}+\epsilon_{o}^{2}+2 \epsilon_{o}\left(4 \epsilon_{e}-3 \epsilon\right)>0,
$$

therefore, its roots are real. Since $\epsilon-2 \epsilon_{e}<0$, these roots have different signs. Let us denote the positive root as

$$
t_{1}=\frac{\epsilon_{o}-\epsilon+\sqrt{D}}{2 \epsilon_{o}} .
$$

The expression $-F_{2} / F_{4}$ will be positive if and only if

$$
0<t<t_{1} \text {. }
$$

For the square of quantity $\beta$, calculated from (8) to be positive, it is necessary and sufficient that

$$
t<\sqrt{\frac{\epsilon_{e}}{\epsilon_{o}}}
$$

was valid. For the square of quantity $q$, calculated from (7) to be positive, it is necessary and sufficient that the condition

$$
q_{o}^{2}>\epsilon-\epsilon_{o}
$$

or

$$
\frac{-F_{2}(1, t)}{F_{4}(1, t)}>\epsilon-\epsilon_{o}
$$

was satisfied.

In the Dyakonov case $-F_{2}>0$ and provided that the inequality (13) is fulfilled, the expression $F_{4}>0$, therefore inequality (14) can be rewritten as

$$
-F_{2}(1, t)>\left(\epsilon-\epsilon_{o}\right) F_{4}(1, t)
$$


or

$$
\left(\epsilon_{o} t+\epsilon_{e}\right)^{2}>-\left(\epsilon_{o} t^{2}+\left(\epsilon-\epsilon_{o}\right) t+\epsilon-2 \epsilon_{e}\right)\left(\epsilon_{o} t+\epsilon\right)(t+1) .
$$

For the square of the value $\gamma$ calculated by the formulas (9) to be positive, it is necessary and sufficient that

$$
\left(1-t^{2}\right) q_{o}^{2}>\epsilon_{e}-\epsilon_{o}
$$

or

$$
\left(1-t^{2}\right)\left(\epsilon_{o} t+\epsilon_{e}\right)^{2}>-\left(\epsilon_{o} t^{2}+\left(\epsilon-\epsilon_{o}\right) t+\epsilon-2 \epsilon_{e}\right)\left(\epsilon_{o} t+\epsilon\right)(t+1) .
$$

To summarize what has been said: the solution of system (5) obtained by the parametric formulas written out falls into the region (11) if and only if

1) the parameter $t$ belongs to the interval

$$
0<t<\min \left(t_{1}, \sqrt{\frac{\epsilon_{e}}{\epsilon_{o}}}\right)
$$

2) two inequalities (15) and (16) are satisfied, and

3) the last equation of system (5) is valid for the choice of the radical principal value.

The fulfillment of these conditions can be checked in the Sage system.

Example. Let $\epsilon_{o}=2, \epsilon=3, \epsilon_{e}=5$. Figure 1 presents a plot of $q_{o}$ (black line) at the values of parameter $t$, taken from the interval (17), dash lines indicate the boundaries of the domain of $t, q_{o}$ variation, determined by inequalities (15) and (16). The restrictions of $t$ are seen to automatically provide the fulfillment of conditions for $q_{o}$. There is the only point $t=1 / 2$, where the plot touches the lower boundary. Just at this point the expression for $q$ changes its sign. Figure 2 presents the plot of the right-hand side of the last equation for the chosen sign ' + ': up to the point $t=1 / 2$ we get a solution, and after this point not. Therefore, the solution satisfies the condition $q>0$ only at $0<t<1 / 2$.

For these values $\beta$ and $\gamma$ were calculated which appeared to be real-valued. For clarity, figure 3 presents a plot of dependence of $t$ on the angle $u$, expressed as

$$
\frac{\gamma}{\beta}=\frac{k_{z}}{k_{y}}=\tan u
$$

It turns out that the surface wave arises only in the directions

$$
\vec{k}_{\perp}=\left(0, k_{y}, k_{z}\right)^{T},
$$

within a narrow range of angles $38-45^{\circ}$.

This example shows that 1) inequalities (15) and (16) are fulfilled in the entire considered interval of parameter $t$ variation, and 2) upon the choice of principal value for the radical our explicit formulae yield a solution to system (5) only up to the point where the plot of $q_{o}$ touches the lower boundary of its corridor. We believe that these assertions can be proved in a purely algebraic way. 


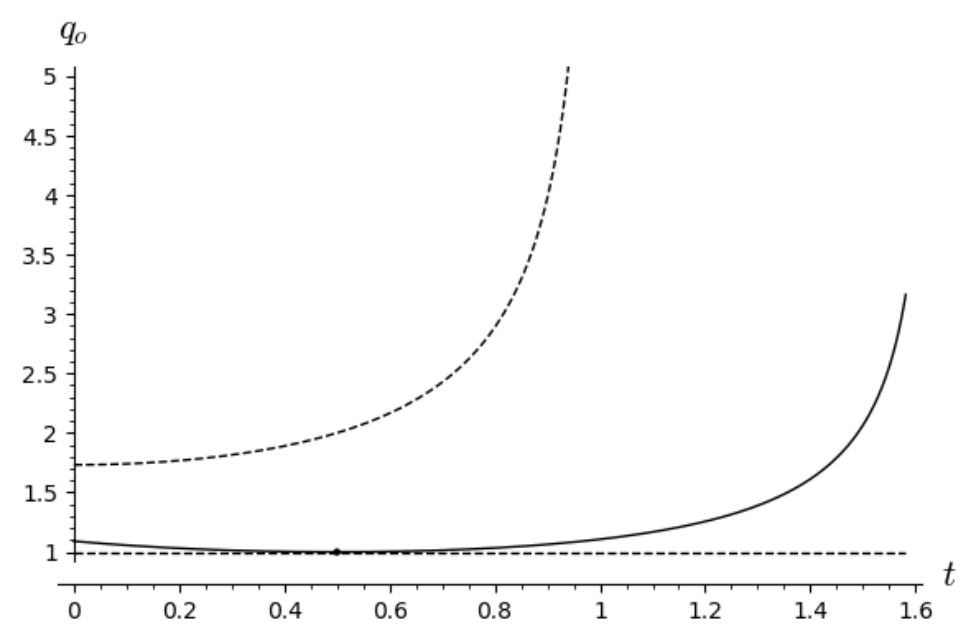

Figure 1. Plot of $q_{o}$ (black line), dash lines indicate the boundaries of the domain of $t, q_{o}$ variation

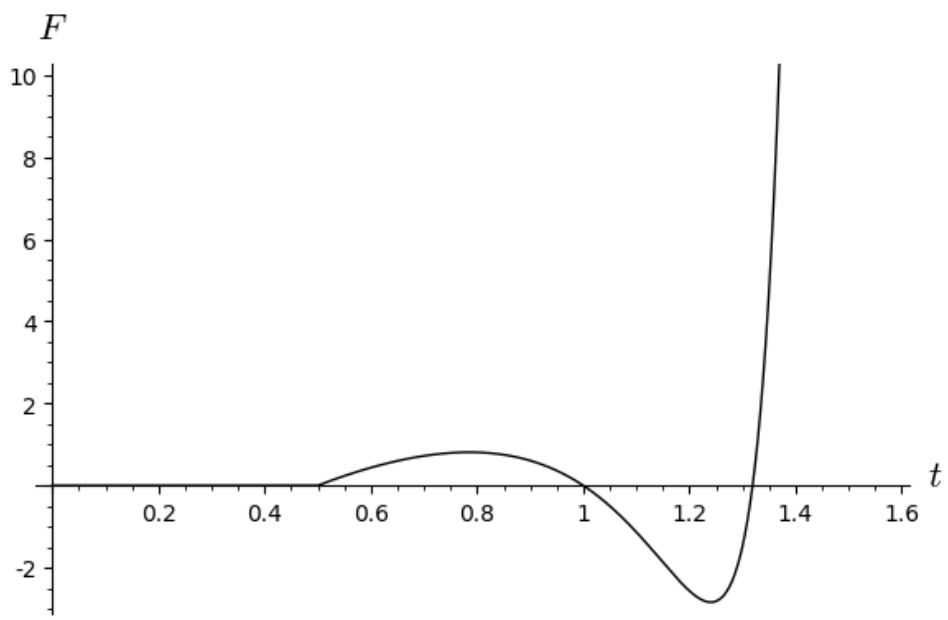

Figure 2. Plot of the left-hand side of the last of equations (5)

\section{Results of computer experiments}

We conducted a series of computer experiments, the results of which are given in the table 1. Based on them, we can draw the following conclusions:

1) the greater the difference between $\epsilon$ and $\epsilon_{e}$, and the smaller it is between the values $\epsilon_{o}$ and $\epsilon$, the greater the half-width of the interval for changing the angle $u$ (see the table 1 cases numbered 12 and 13);

2 ) the smaller the difference between $\epsilon$ and $\epsilon_{e}$, and the larger it is between the values $\epsilon_{o}$ and $\epsilon_{e}$, the smaller the half-interval of the angle $u$ (see the table 1 cases numbered 14 and 15); 


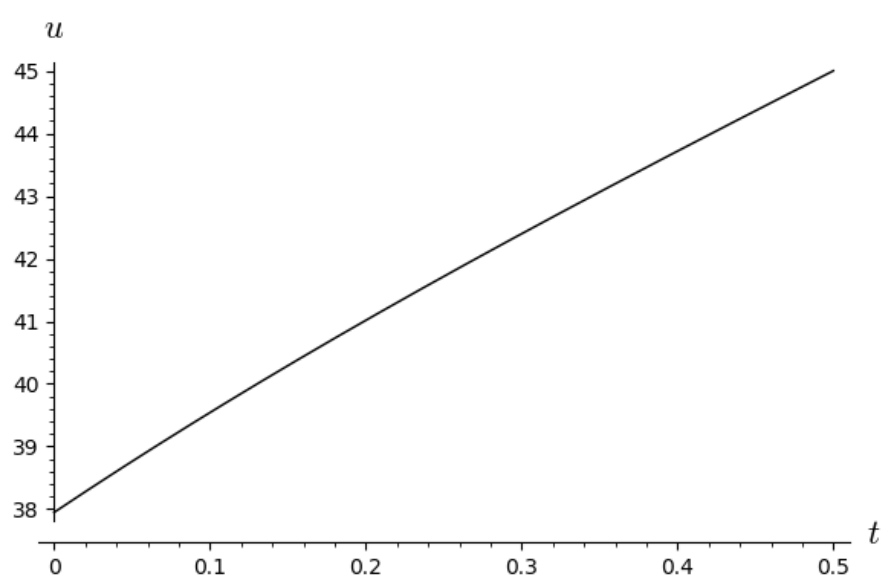

Figure 3. Plot of $u$, the angle is in degrees

$3)$ if the difference between the values $\epsilon_{o}, \epsilon, \epsilon_{e}$ remains constant, and the values themselves increase, then the half-interval of the angle $u$ variation, in which surface waves exist, decreases (see the table 1, cases numbered 4 , $16,17,18)$;

4) if the difference between the values of $\epsilon_{o}, \epsilon, \epsilon_{e}$ is increased by the same amount, the half-interval of $u$ variation is practically unchanged (see the table 1, cases 19-21).

These conclusions are awaiting theoretical substantiation.

We failed to find a simple relationship between the position of the mean angle $u$ and the values of $\epsilon_{o}, \epsilon, \epsilon_{e}$, while the position of the mean angle $u$ itself changes quite noticeably.

To test our approach, we looked at several non-Dyakonov cases where one of the (10) inequalities is violated. As expected, no solutions were found in these cases.

\section{Conclusion}

Investigation of the region of existence of the Dyakonov surface wave at the isotropic-anisotropic interface is reduced to a system of algebraic equations and inequalities. We managed to solve this system of equations in radicals in the Sage computer algebra system. At the same time, the inequalities were only partially investigated analytically. On this basis, it was possible to carry out a series of computer experiments clarifying further directions of research.

\section{Acknowledgments}

The author thanks Prof. L. A. Sevastyanov and M. D. Malykh (RUDN University) for setting an interesting problem and permanent attention to his work. All calculations presented in the article were performed in the Sage system Sage. The publication was supported by the RUDN University Strategic Academic Leadership Program. 
Table 1

Results of computer experiments

\begin{tabular}{|c|c|c|c|c|c|}
\hline No. & $\epsilon_{o}$ & $\epsilon$ & $\epsilon_{e}$ & Mean angle $u$, deg. & $\begin{array}{l}\text { Half-interval of } \\
\text { angle } u \text { varia- } \\
\text { tion, deg. }\end{array}$ \\
\hline 1 & 1 & 2 & 3 & $27-34$ & 3.5 \\
\hline 2 & 2 & 3 & 5 & $38-45$ & 3.5 \\
\hline 3 & 2.5 & 3.5 & 5.5 & $40.5-47$ & 3.25 \\
\hline 4 & 3 & 4 & 6 & $42.4-48.5$ & 3.05 \\
\hline 5 & 3 & 4 & 10 & $45.6-53.8$ & 4.1 \\
\hline 6 & 3.0001 & 3.001 & 3.01 & $72.426-72.455$ & 0.0145 \\
\hline 7 & 3 & 3.01 & 3.02 & $44.911-45$ & 0.0445 \\
\hline 8 & 3 & 4 & 4.001 & $1.57-1.76$ & 0.095 \\
\hline 9 & 3.9 & 4 & 4.1 & $44.299-44.96$ & 0.3305 \\
\hline 10 & 3.9 & 4 & 4.001 & $5.649-5.71$ & 0.0305 \\
\hline 11 & 3.99 & 4 & 4.001 & $17.522-17.548$ & 0.013 \\
\hline 12 & 2 & 7 & 55 & $13.2-19.2$ & 3 \\
\hline 13 & 2 & 2.1 & 80 & $45.47-56$ & 5.265 \\
\hline 14 & 2 & 54 & 55 & $1.5-2.22$ & 0.36 \\
\hline 15 & 2 & 79.9 & 80 & $0.3275-0.4825$ & 0.155 \\
\hline 16 & 13 & 14 & 16 & $51.5-53.58$ & 1.04 \\
\hline 17 & 33 & 34 & 36 & $53.59-54.46$ & 0.435 \\
\hline 18 & 133 & 134 & 136 & $54.446-54.78$ & 0.167 \\
\hline 19 & 20 & 40 & 60 & $27-34$ & 3.5 \\
\hline 20 & 20 & 50 & 80 & $23-30$ & 3.5 \\
\hline 21 & 20 & 60 & 100 & $20.2-27.2$ & 3.5 \\
\hline
\end{tabular}

\section{References}

[1] F. N. Marchevskii, V. L. Strizhevskii, and S. V. Strizhevskii, "Singular electromagnetic waves in bounded anisotropic media," Sov. Phys. Solid State, vol. 26, p. 857, 1984.

[2] M. I. Dyakonov, "New type of electromagnetic wave propagating at an interface," Sov. Phys. JETP, vol. 67, p. 714, 1988. 
[3] O. Takayama, L.-C. Crasovan, S. Johansen, D. Mihalache, D. Artigas, and L. Torner, "Dyakonov surface waves: a review," Electromagnetics, vol. 28 , pp. 126-145, 2008. DOI: 10.1080/02726340801921403.

[4] J. A. Polo Jr. and A. Lakhtakia, "A Surface Electromagnetic Waves: a Review," Laser 83 Photonics Reviews, vol. 5, pp. 234-246, 2011. DOI: 10.1002/lpor. 200900050.

[5] O. N. Bikeev and L. A. Sevastianov, "Surface electromagnetic waves at the interface of two anisotropic media," RUDN Journal of Mathematics, Information Sciences and Physics, vol. 25, no. 2, pp. 141-148, 2017, in Russian. DOI: 10.22363/2312-9735-2017-25-2-141-148.

[6] O. N. Bikeev, K. P. Lovetskiy, N. E. Nikolaev, L. A. Sevastianov, and A. A. Tiutiunnik, "Electromagnetic surface waves guided by a twist discontinuity in a uniaxial dielectric with optic axis lying in the discontinuity plane," Journal of Electromagnetic Waves and Applications, vol. 33, no. 15, pp. 2009-2021, 2017. DOI: 10.1080/09205071.2019.1655486.

[7] O. Takayama, L.-C. Crasovan, D. Artigas, and L. Torner, "Observation of Dyakonov surface waves," Physical Review Letters, vol. 102, p. 043 903, 2009. DOI: 10.1103/PhysRevLett.102.043903.

[8] O. Takayama, D. Artigas, and L. Torner, "Lossless directional guiding of light in dielectric nanosheets using Dyakonov surface waves," Nature Nanotech, vol. 9, pp. 419-424, 2014. DOI: 10.1038/nnano.2014.90.

[9] D. Cox, J. Little, and D. O'Shea, Ideals, varieties, and algorithms, 2nd ed. Springer, 1997.

\section{For citation:}

O.K.Kroytor, Investigation of the existence domain for Dyakonov surface waves in the Sage computer algebra system, Discrete and Continuous Models and Applied Computational Science 29 (2) (2021) 114-125. DOI: 10.22363/2658-4670-2021-29-2-114-125.

\section{Information about the authors:}

Kroytor, Oleg K. - Postgraduate of Department of Applied Probability and Informatics of Peoples' Friendship University of Russia (RUDN University); (e-mail: kroytor_ok@pfur.ru, phone: +7(495)9550927, ORCID: https://orcid.org/0000-0002-5691-7331) 
УДК 519.872:519.217

PACS 07.05.Tp, 02.60.Pn, 02.70.Bf

DOI: $10.22363 / 2658-4670-2021-29-2-114-125$

\title{
Исследование области существования поверхностных волн Дьяконова в системе компьютерной алгебры Sage
}

\author{
О. К. Кройтор \\ Российский университет дружбы народов \\ ул. Миклухо-Маклал, д. 6, Москва, 117198, Россия
}

Рассмотрены поверхностные электромагнитные волны (волны Дьяконова), распространяющиеся вдоль плоской границы раздела изотропного вещества с постоянной диэлектрической проницаемостью, и анизотропного кристалла, тензор диэлектрической проницаемости которого имеет ось симметрии, направленную вдоль границы раздела. Хорошо известно, что вопрос о существовании таких поверхностных волн сводится к вопросу о существовании решения некоторой системы алгебраических уравнений и неравенств. В настоящей работе эта система исследована в системе компьютерной алгебры Sage.

Техника исключительных идеалов, встроенная в Sage, позволила описать решение системы алгебраических уравнений параметрически при помощи одного параметра, причём все исходные величины выражаются через этот параметр при помощи радикалов. Оставшиеся неравенства удалось исследовать аналитически лишь частично. Для полного исследования разрешимости системы уравнений и неравенств предложен и реализован в Sage символьно-численный алгоритм, представлены результаты компьютерных экспериментов. На основе результатов экспериментов были сделаны выводы, которые требуют дальнейшего теоретического обоснования.

Ключевые слова: поверхностные волны, волны Дьяконова, электромагнитные волны, компьютерная алгебра, Sage 